\section{Ruptured Ectopic Tubal Gestation: An Unsolvable Conundrum on Imaging!}

Sir,

An ectopic pregnancy (EP) is a condition in which a fertilised ovum implants in a location other than normal endometrial cavity. It is diagnosed by estimation serum beta-HCG level and transvaginal ultrasonography. Despite being a common condition, the complex radiologic appearance of ruptured EP has rarely been described in the literature. We share a unique case of ruptured EP on MR imaging.

A 25-year female, P0 G1, presented to Gynaecology/ Obstetrics Department with 2-month amenorrhea and lower abdominal pain. The investogram specifically showed serum $\beta$-HCG levels within normal limits (42.89 $\mathrm{mIU} / \mathrm{ml}$ ). Ultra-sonography showed a large pelvic indeterminate mass with normal uterus. Doppler study showed no significant flow, which was inconclusive for EP. CT scan showed a complex, heterogeneous, iso-to hyper-dense, solid / cystic, minimally enhancing, mass in the cul-de-sac involving bilateral adnexa. Foci of calcifications were noted within it. The MRI examination of the pelvis revealed a large $11 \times 9 \times 11 \mathrm{~cm}$ in APxTRxCC, thick walled T1, T2 hyper-intense complex haemorrhagic mass with fluid-fluid levels and post-contrast rim enhancement. Normal ovaries were seen (Figure 1). No free fluid in the pelvis was seen. At this point, we raised the differentials of haematosalpinx, tubo-ovarian complex, and tubal endometriosis. The patient was vitally stable, so proceeded to salpingectomy; and the sample was sent for histopathology, which showed wall of fallopian tube distended by blood and fibrin with few hyalinised chorionic villi; compatible with ruptured ectopic tubal gestation. We did retro-spective analysis of MRI, which showed fallopian tube defect at left adnexa (Figure 2). It was hypothesised that hematoma in the pelvis was walled off by thick wall and did not lead to hemoperitoneum/free fluid. The patient underwent normal postoperative recovery and survived.

Tamai et al. described the imaging and histopathological features of EP. 1 They highlighted that recognition of wall enhancement of dilated tubal structure raises possibility of tubal pregnancy, which reflected increased vascularity of the tubal wall following implantation. On MRI, gestational sac is seen as a cyst (fluid-filled structure) with surrounding hematoma of distinct low intensity on

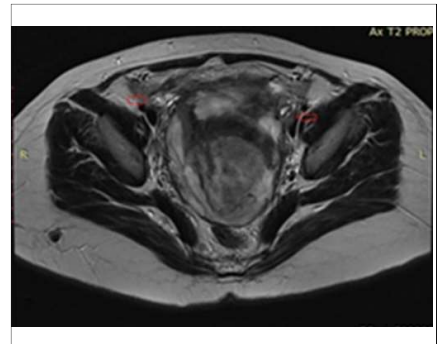

Figure 1: Contiguous axial T2-weighted sections of the pelvis showing large pelvic mass with separate ovaries (arrows).

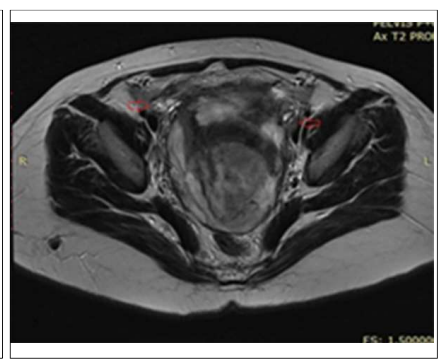

Figure 2: Contiguous axial T1-weighted image of the pelvis showing large pelvic mass rupture of the left fallopian tube (arrows).

T2-weighted images, and high signal intensity on T1weighed images. The placental tissue appeared as large heterogeneously enhancing mass or papillary solid component within the dilated tube. On laparoscopy, hematosalpinx was seen with trophoblastic tissue. ${ }^{1}$ The serum $\beta$-HCG levels were normal in our case. Murray et al. also documented that EP may present with rising, falling or plateau $\beta-\mathrm{HCG}$ levels; and serial measurement is used to confirm fetal viability rather than to identify EP. ${ }^{2}$ After exuberant literature search, only a single case on MR imaging, describing hematosalpinx and ruptured tubal pregnancy, was found that showed a T1- and T2hyperintense complex cystic solid mass, in close contiguity with fallopian tube. ${ }^{3}$ The findings at surgery confirmed a ruptured tubal EP.

This case highlights the need for radiologists to consider always the possibility of EP in the setting of pelvic mass in a woman of child-bearing age.

\section{CONFLICT OF INTEREST:}

Authors declared no conflict of interest.

\section{AUTHORS' CONTRIBUTION:}

$\mathrm{MH}$ : Involved in study concepts, designing and editing manuscript.

SM: Contributed in manuscript preparation and literature search.

NM: Involved in surgical procedures of the case and provision of histopathological results.

\section{REFERENCES}

1. Tamai K., Koyama T, Togashi K. MR. features of ectopic pregnancy. Eur Radiol 2007; 17:3236-46.

2. Murray $\mathrm{H}$, Baakdah $\mathrm{H}$, Bardell $\mathrm{T}$, Tulandi $\mathrm{T}$. Diagnosis and treatment of ectopic pregnancy. Can Med Assoc J 2005; 173: 905-12.

3. Parker RA, Yano M, Tai AW, Friedman M, Narra VR, Menias CO. MR imaging findings of ectopic pregnancy: A pictorial review. Radiographics 2012; 32:1445-60. 
Mahnoor Hafeez ${ }^{1}$, Sohbia Munir ${ }^{1}$ and Nazia Munir ${ }^{2}$

1 Dow Institute of Radiology, Dow University of Health Sciences (DUHS), Karachi, Pakistan

2 Department of Gynecology and Obstetrics, Dow University of Health Sciences (DUHS), Karachi, Pakistan.
Correspondence to: Dr. Mahnoor Hafeez, Dow Institute of Radiology, Dow University of Health Sciences (DUHS),

Karachi, Pakistan

E-mail:mahnoor.hafeez@yahoo.com

Received: January 05, 2019; Revised: April 26, 2019;

Accepted: May 03, 2019

.......... 